\title{
Childhood Classic Hodgkin Lymphoma
}

National Cancer Institute

\section{Source}

National Cancer Institute. Childhood Classic Hodgkin Lymphoma. NCI Thesaurus. Code C69142.

A classic Hodgkin lymphoma that occurs during childhood. 